\title{
Framework Conceitual de apoio ao design de Museus Virtuais com foco em Interfaces Naturais
}

\author{
Guilherme Corredato Guerino $^{1}$; Breno Augusto Guerra Zancan² ${ }^{2}$ Tatiany Xavier de Godoi ${ }^{1}$; \\ Daniela de Freitas Guilhermino Trindade ${ }^{2}$, José Reinaldo Merlin², Ederson Marcos Sgarbi \\ Carlos Eduardo Ribeiro², Tércio Weslley Sant'Anna de Paula Lima \\ ${ }^{1}$ Universidade Federal do Paraná, \\ Curitiba - PR, Brasil \\ ${ }^{2}$ Universidade Estadual do Norte do Paraná \\ Bandeirantes - PR, Brasil \\ gcguerino@inf.ufpr.br; brenozancan@gmail.com; txgodoi@inf.ufpr.br; danielaf@uenp.edu.br, \\ merlin@uenp.edu.br, sgarbi@uenp.edu.br,biluka@uenp.edu.br, terciowspl@gmail.com
}

\section{RESUMO}

O Museu Virtual é um importante instrumento que permite o acesso a obras, muitas vezes, inacessíveis devido à localização física dos museus tradicionais. No entanto, observa-se que os museus virtuais apresentam problemas que afetam diretamente sua usabilidade. Assim, neste artigo é proposto um framework conceitual que fornece um modelo conceitual e diretrizes relacionadas às Interfaces Naturais de Usuários para apoiar o design de Museus Virtuais. Para verificar a sua aplicabilidade, o framework apoiou o desenvolvimento de um Museu Virtual para salvaguardar a história de uma cidade. O museu virtual foi disponibilizado à um conjunto de usuários que forneceram um feedback sobre a sua experiência. A partir dos relatos, observou-se que, seguindo os eixos e diretrizes propostos pelo VMNUI, foi possível criar um museu virtual que proporcionasse uma boa experiência de navegação, interação e comunicação.

\section{Palavras-chave dos autores}

Museu Virtual; framework conceitual; interface natural; usabilidade; comunicabilidade; interatividade.

\section{ACM Classification Keywords}

Natural User Interface; Usability.

\section{INTRODUÇÃO}

O Museu Virtual é um importante meio de comunicação que permite preservar informações históricas que muitas vezes são perdidas. Segundo [1], um Museu Virtual pode ser definido como um acervo de conteúdo digital que é

\footnotetext{
Permission to make digital or hard copies of all or part of this work for personal or classroom use is granted without fee provided that copies are not made or distributed for profit or commercial advantage and that copies bear this notice and the full citation on the first page. Copyrights for components of this work owned by others than the author(s) must be honored. Abstracting with credit is permitted. To copy otherwise, or republish, to post on servers or to redistribute to lists, requires prior specific permission and/or a fee. Copyright 2018 SBC.
}

IHC 2018, Anais Estendidos do XVII Simpósio Brasileiro sobre Fatores Humanos em Sistemas Computacionais Outubro 22-26, 2018, Belém, Brasil

Artigos Internacionais disponibilizados na web. Se constitui como um ambiente, que rompe a barreira de espaço e tempo e que pode, segundo [2], facilitar a recepção informativa, pedagógica e estética proporcionada por museus convencionais.

Algumas falhas de usabilidade que desestimulam o público a explorar os conteúdos foram identificadas por [3], que analisaram 36 museus virtuais. Nesse contexto, a Interface Natural pode se apresentar como uma forma de minimizar estes problemas, pois, segundo [4], por meio dela o usuário pode interagir com conteúdos digitais utilizando movimentos, gestos, sons e toques, os quais são mais intuitivos que o uso de dispositivos de controle artificial.

Face a este cenário, observou-se a relevância de propor modelos que apoiem os designers de ambientes virtuais na criação de espaços que proporcionem boa interatividade, intuitividade e melhor usabilidade. Assim, este artigo apresenta um framework conceitual de apoio à criação de museus virtuais com foco nas características das interfaces naturais. Um maior detalhamento do framework proposto foi apresentado em [5].

\section{VMNUI: FRAMEWORK CONCEITUAL DE APOIO AO DESIGN DE MUSEUS VIRTUAIS COM FOCO EM INTERFACES NATURAIS}

A partir dos diagnósticos efetuados, com base nos principais fatores do design de sistemas interativos proposto por [6] e no trabalho de [7], foi desenvolvido o framework conceitual VMNUI (Virtual Museums with Natural User Interface).

O framework é composto pelo modelo conceitual (Figura 1) e as diretrizes propostas, com ênfase nas interfaces naturais, buscando apoiar a criação de museus virtuais tridimensionais. No modelo são apresentados os diferentes papéis que as pessoas podem assumir no Museu Virtual, além dos componentes que podem ser utilizados para divulgar o conteúdo. 


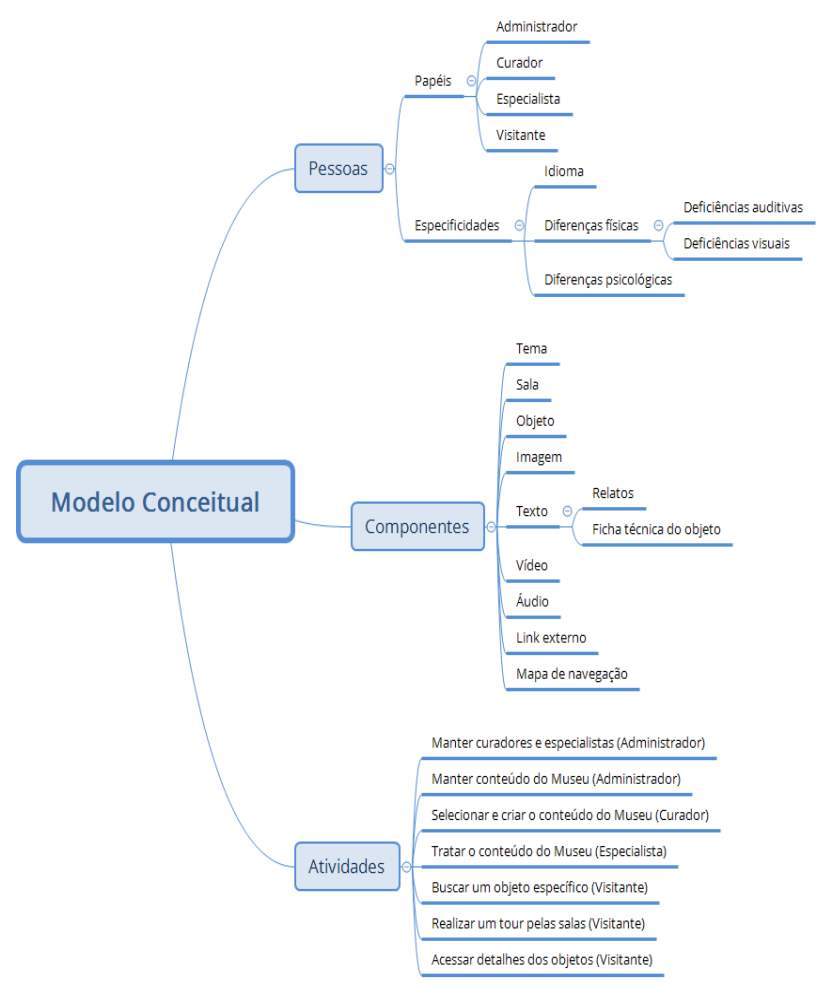

Figura 1. Modelo Conceitual do framework VMNUI [4].

Ainda, são expostas as atividades que cada pessoa pode realizar em Museu Virtual. O VMNUI é composto também por algumas diretrizes que tem como função auxiliar os desenvolvedores de museus virtuais na construção de ambientes mais intuitivos e agradáveis. Um resumo das diretrizes é apresentado na Tabela 1 .

\begin{tabular}{c|c} 
Diretriz & Definição \\
\hline Idioma & Opção de tradução \\
\hline Diferenças físicas & Atentar para as deficiências \\
\hline Imagens $360^{\circ}$ & $\begin{array}{c}\text { Rotacionar o ambiente em todos } \\
\text { os ângulos }\end{array}$ \\
\hline Função 3D & $\begin{array}{c}\text { Rotacionar as obras } \\
\text { horizontalmente }\end{array}$ \\
\hline Touchscreen & Utilizar a função touchscreen \\
\hline Voz & Permitir o comando por voz \\
\hline $\begin{array}{c}\text { Fisiologia e } \\
\text { cinestesia }\end{array}$ & $\begin{array}{c}\text { Evitar movimentos difíceis e } \\
\text { ações repetitivas }\end{array}$ \\
\hline $\begin{array}{c}\text { Disposição das } \\
\text { informações }\end{array}$ & $\begin{array}{c}\text { Promover boa visibilidade de } \\
\text { todos elementos da interface }\end{array}$
\end{tabular}

Tabela 1. Diretrizes do framework VMNUI

As NUI's, segundo [8], surgem com o objetivo de utilizar habilidades diárias do usuário para a interação com o conteúdo. Assim, é possível que os usuários tirem proveito das habilidades adquiridas no seu cotidiano e isso contribui para que o tempo de aprendizagem para a utilização da interface natural seja menor.

\section{CONSIDERAÇÕES FINAIS}

O Museu Virtual é uma alternativa para muitos indivíduos que desconhecem a existência de museus físicos, ou não têm a motivação necessária para fazer uma visita, por conta de diversas razões que podem dificultar o seu acesso.

Buscando verificar a sua aplicabilidade, o VMNUI apoiou o desenvolvimento de um Museu Virtual para salvaguardar a história da cidade de Bandeirantes - Paraná/Brasil. Um grupo de pessoas teve acesso à primeira versão do Museu, e puderam relatar a sua experiência no ambiente. Algumas questões foram apontadas, dentre elas a possibilidade de acesso aos textos das imagens e a necessidade de inclusão de alguns botões para melhorar a navegação. Contudo, a partir dos relatos, observou-se que, seguindo os eixos e diretrizes propostos pelo VMNUI, foi possível criar um museu virtual que proporcionasse, no geral, uma boa experiência de navegação.

\section{REFERÊNCIAS}

1. Moura, I. B. G., Mendes Neto, F. M., Sousa, P. S. M. 2012. Utilização do Framework Jade no Desenvolvimento de um Museu Virtual 3D com Recomendação Personalizada de Conteúdo. Exacta, v. 5, pp. 83-97, Belo Horizonte.

2. Muchacho, R. 2005. Museus virtuais: A importância da usabilidade na mediação entre o público e o objecto museológico. Livro de actas do $4^{\circ}$ Congresso da Associação Portuguesa de Ciências da Comunicação, pp. 1540-1547, Portugal.

3. Marty, P. F., Twidale, M. B. 2004. Lost in gallery space: A conceptual framework for analyzing the usability flaws of museum Web sites. First Monday, v. $9, \mathrm{n}^{\circ} 9$.

4. Arrais, M., Grossi, M. G. R., Martins, A. R. G. 2012. Interface Natural do Usuário: Aplicações para a Inovação do Ensino a Distância com o Uso do Microsoft Kinect ${ }^{\circledR}$. In: $18^{\circ}$ Congresso Internacional de Educação a Distância (ABED), v.18. São Paulo.

5. Guerino, G., Zancan, B. A. G., Godoi, T. X., Trindade, D. F. G., Merlin, J. R., Sgarbi, E. M., Ribeiro, C. E., Lima, T. W. S. P. 2018. Conceptual Framework for Supporting the Creation of Virtual Museums with Focus on Natural User Interfaces. In: Marcus A., Wang W. (eds) Design, User Experience, and Usability: Users, Contexts and Case Studies. DUXU 2018. Lecture Notes in Computer Science, Vol10920. Springer, Cham.

6. Barbosa, S. D. J., Silva, B. S. 2010. Interação HumanoComputador. 1st edn. Rio de Janeiro: Campus.

7. Schneider, E. I. 2012. Uma contribuição aos Ambientes Virtuais de Aprendizagem (AVAs) suportados pela Teoria da Cognição Situada (TCS) para pessoas com deficiência auditiva. Santa Catarina.

8. Blake, J. 2011. Natural User Interfaces in .Net. 1st edn. Nova York. 